Eighty countries have adopted the WHO's list of 200 or so essential drugs. Of these, those with the longest experience with a limited list (Papua New Guinea, Mozambique, Sri Lanka, Kenya, and Tanzania) are beginning to evaluate the medical, economic, and political implications of this policy. In collaboration with Unicef and other organisations the WHO has succeeded in decreasing the prices of drugs to the extent that all those needed for primary health care should cost no more than about $35 \mathrm{p}$ a head a year. Speaking on behalf of the WHO, E Lauridsen said that countries that paid over the odds for their drugs-sometimes 10 times over-did so not through ignorance of the correct sources of cheap, high quality drugs but for political motives of their own.

Two further meetings are scheduled for next month. Firstly, the European parliament is going to debate the draft report prepared by Mrs M Banotti on the export of drugs from the European Community to the Third World. The key recommendation is that drugs banned, withdrawn, severely restricted, or not licensed for sale within the EEC should not be exported to developing countries-unless the health authorities have requested it in the full knowledge of the controls that have been applied in Europe. This is a welcome initiative for it could do a lot to eliminate the double standards that are being applied with respect to safety. Secondly, 18 motions have been put forward for discussion at the next annual representative meeting of the British Medical Association (23-26 June) on the problems of supplying developing countries with essential drugs, ways to persuade the pharmaceutical industry not only to respond to this need but also to devote more money towards the research and development of drugs to treat tropical diseases, and stopping the supply of irrational and inessential drugs.

In response to this mounting pressure the pharmaceutical industry has retaliated. The Association of the British Pharmaceutical Industry has described Health Action International's findings as alarmist, re-emphasising that the association supports the establishment of effective national medicines policies and regulatory bodies and the extension of the WHO's certification scheme to ensure the quality and availability of full information about medicines world wide. What the industry is clearly not prepared to accept, however - and rightly - is the role of scapegoat for the Third World's health problems. Its arguments are well rehearsed: developing countries need to increase their literacy rates, establish systems of political and social order, improve transport and communication systems, provide clean water supplies and effective sanitation, improve local agricultural economies, and build up effective primary and secondary health care services. ${ }^{3}$ Improving the health care infrastructure by these means is, it argues, more important than imposing rigid limited lists.

No one would dispute that these measures are vital, or that the experience of those developing countries with limited lists should be evaluated very carefully, for there are lessons to be learnt. In Bangladesh, for example, where there has been a limited list for nearly four years, the policy has been successful but it has created some difficulties. ${ }^{45}$ Nevertheless, surely the industry should shoulder much of the blame for the current inappropriate and excessive use of medicines in developing countries. And it must produce more convincing arguments to support its thesis that the voluntary code of practice of the International Federation of Pharmaceutical Manufacturers Association (designed to protect consumers against unethical drug promotion) is working. It is encouraging to see that the industry is showing concern, but it must show its critics that this is more than skin deep.

Assistant editor, $B M \mathcal{F}$

TESSA RICHARDs

1 Chetlev A, Gilbert D. Problem drugs. The Hague: Health Action International, 1986.

Rhode JE. Selective primary health care: strategies for control of disease in the developing world XV. Acute diarrhoea. Rev Infect Dis 1984;6:840-54.

aylor D. Pharmaceutical briefing. Development, health and the need for pharmaceuticals. London:

Association of the British Pharmaceutical Industry, 1986

Islam SMD. Drug policy in the Third World. Lancet 1985; ii:46-7.

5 Ahmed M. Drug policy in the Third World. Lancet 1985;ii:47.

\section{Progress towards a new pertussis vaccine}

In 1975 the pertussis vaccine used in Japan was withdrawn $\vec{\circ}$ after the sudden deaths of two recently vaccinated infants. The vaccination programme was later reinstated, but public $\stackrel{\circ}{\circ}$ confidence was not restored, and the poor uptake led to a $\frac{5}{3}$ swift resurgence of whooping cough. ${ }^{1}$ In Britain, too, i pertussis epidemics returned rapidly when acceptance fell in the mid-1970s after adverse publicity about the safety of the of vaccine. The need for routine pertussis immunisation is now no longer questioned in Britain and interest is currently $\vec{\omega}$ focused on the development of a purified vaccine containing $\stackrel{+}{\infty}$ identifiable protective antigens.

Whole cell vaccine consists of a suspension of killed $N$ Bordetella pertussis organisms. It is, therefore, a mixture of antigens, some of which-while essential for protection- $\underset{<}{\stackrel{0}{2}}$ may also produce reactions. The aim of current research is to $\vec{\oplus}$ identify and purify the protective antigens from the whole 8 cell and inactivate those which are toxic - and so to assemble a component vaccine which gives good protection with minimal reactogenicity. The main toxic component of the organism is thought to be lymphocytosis promoting factor. When injected into animals this factor causes lymphocytosis, $\frac{\mathbb{Q}}{\triangle}$ hypoglycaemia, histamine sensitisation, and other effects. $\overrightarrow{\vec{O}}$ There is general agreement that lymphocytosis promoting $\frac{3}{3}$ factor, suitably treated to destroy its toxic activity while retaining its immunogenicity, should be included in a vaccine to protect against the toxic effects of the infection. ${ }^{2} \mathrm{~A}$ second component thought to be important for protection is filamentous haemagglutinin, a surface antigen which prob- $\dot{0}$ ably plays a part in the initial colonisation phase of the infection and appears to be non-toxic. ${ }^{23}$ Opinion about the $\frac{\delta}{\beta}$ importance of serotype specific agglutinogens is divided despite both laboratory and epidemiological evidence sug- 을 gesting that these antigens are concerned in protection. ${ }^{45} \mathrm{~N}$ Several other components of $B$ pertussis have been identified, $D$ and some are known to induce antibodies in recipients of whole cell vaccine, ${ }^{6}$ but their importance in protection is $\tilde{N}$ uncertain. There is agreement, however, that a component $\mathrm{\omega}$ vaccine should contain little or no lipopolysaccharide antigen, an endotoxin present not only in $B$ pertussis but in other Gram negative bacteria. ${ }^{2}$

Component vaccines containing variable amounts of filamentous haemagglutinin and inactivated lymphocytosis promoting factor (but with some degree of contamination with other antigens) were introduced in Japan in 1981 for routine use in 2 year olds. ' Laboratory tests showed the vaccines to be less toxic than the whole cell preparations, and they are reported to have produced fewer feverish and local reactions in children. ${ }^{1}$ A clinical protection trial of two Japanese component preparations has started in Sweden, where 
pertussis has become a substantial public health problem since the withdrawal of whole cell vaccine in $1979 .^{7}$ The development of a British component vaccine at the Public Health Laboratory Service Centre for Applied Microbiology and Research is now nearing completion, and clinical trials are being planned under the direction of the Medical Research Council. The background against which the British trials will take place, however, is somewhat different from that in Japan and Sweden. In Britain whole cell vaccine was never withdrawn despite the controversy about the risks and benefits of vaccination. Instead, epidemiological investigations were undertaken to establish the frequency of adverse reactions attributable to the pertussis component of current vaccines. These studies showed only small differences in the frequency of minor symptoms after adsorbed diphtheriatetanus-pertussis and diphtheria-tetanus vaccines. ${ }^{89}$ No difference was observed in the frequency of rare symptoms such as high pitched screaming, collapse, and convulsive episodes between two large cohorts of children given the two vaccines who were followed up prospectively through their course of primary immunisation." The evidence is, therefore, that replacing the whole cell pertussis component of British adsorbed diphtheria-tetanus-pertussis vaccine with acellular antigens is unlikely to result in a large reduction in the frequency of symptoms after vaccination. Plain diphtheriatetanus-pertussis preparations without an aluminium hydroxide adjuvant are still sometimes used in Britain; these have been shown to be much more reactogenic than adsorbed preparations. ${ }^{89}$ Unfortunately, in the Japanese trials an adsorbed component vaccine was compared with a plain whole cell preparation; the greater reactogenicity reported for the latter preparation may largely be due to its lack of adjuvant rather than to the nature of its pertussis component. ${ }^{1}$

The claim that whole cell pertussis vaccine is responsible for characteristic neurotoxic reactions in children has been investigated in several studies in Britain in recent years. ${ }^{9-11}$ No evidence of a "pertussis reaction syndrome" has emerged. A three year nationwide case-control study of acute neurological disorders in childhood did show a small excess in the number of patients who had received diphtheria-tetanuspertussis vaccine within seven days of onset relative to controls. ${ }^{10}$ It also showed a small excess of patients in whom the onset was within seven days of receiving diphtheriatetanus vaccine, though the temporal association was not statistically significant. Clearly a lengthy and extensive epidemiological investigation would be required to establish the relative safety of component vaccines. While evidence of minimal toxicity in laboratory tests and some reduction in the frequency of minor postvaccination symptoms would be reassuring, a component preparation could not be offered as a safer vaccine at the outset. Absence of biologically active lymphocytosis promoting factor and minimal concentrations of lipopolysaccharide in a component vaccine cannot guarantee freedom from rare adverse effects in children, for the mechanism linking vaccination with acute neurological disorders is unknown and does not appear to be specific to vaccines with a pertussis component.

Investigations similar to the large controlled studies carried out in Britain have not been reported from other countries, and whether the safety record of British whole cell vaccine applies to vaccines produced elsewhere remains unknown. ${ }^{910}$ In the United States, the frequency of minor postvaccination symptoms, particularly fever, is reported to be much greater after diphtheria-tetanus-pertussis than diphtheria-tetanus vaccines; the whole cell pertussis component of American vaccines appears therefore to be more reactogenic than that of British preparations. ${ }^{12}$ Differences in the method of manufacture, in the nature and quantity of adjuvant used, and in the number of organisms per dose may result in preparations with differing toxicity and potency, and standardisation of whole cell vaccines has proved difficult. Component vaccines should be easier to standardise, and work is in progress to establish internationally agreed laboratory procedures to define the antigen content of such preparations.

Component vaccines may be less reactogenic than whole cell preparations, but they will be of little use unless they also protect children against whooping cough. Despite growing knowledge, the nature of the host-pathogen interaction in pertussis is still incompletely understood; much of the evidence that certain antigens are protective derives from work in animals, and its relevance to human disease is uncertain. The vaccines developed in Japan are reported to be as effective as plain whole cell preparations, but the evidence comes from a small home contact study of only 36 recipients of component vaccine. ' Whole cell vaccine, despite its full complement of antigens, does not confer solid immunity, ${ }^{13}$ and protection from component preparations might be even less complete if they lacked essential antigens. The evidence that agglutinogens are concerned in protection has been considered in Britain, and the Centre for Applied Microbiology and Research vaccine, unlike component preparations being produced elsewhere, will contain defined amounts of agglutinogens 2 and 3 in addition to filamentous haemagglutinin and inactivated lymphocytosis promoting factor. ${ }^{45}$ The change in prevalent serotype after the introduction of vaccination in Britain and the recent reversion when immunisation rates declined provides further evidence that protection is, at least in part, serotype specific. ${ }^{14}$

British workers are also studying the part played by mucosal immunity in protection, ${ }^{2}$ as there is evidence that the current vaccine is less effective in preventing colonisation of the respiratory tract than symptoms of disease. ${ }^{13}{ }^{15}$ Measurement of pertussis specific antibodies in nasal secretions as well as in serum should, therefore, be included in immunogenicity trials of component vaccines in Britain. Since the antigen content of these preparations may be increased without necessarily increasing reactogenicity one aim of British workers is to produce a vaccine which gives more solid immunity than the conventional whole cell preparation. ${ }^{2}$ This would allow improved control of whooping cough and might offer the additional advantage of requiring only a two dose schedule.

The relative importance of the putative protective antigens included in component vaccines and the dose, schedule, and route of administration required to achieve an optimal effect can be determined only by careful trials of immunogenicity, reactogenicity, and clinical protection. These will take time to organise and complete. Meanwhile clinicians should remember that the whole cell preparations in use in Britain are of low reactogenicity and that their ability to control whooping cough is proved. Despite the rare association between vaccination and acute neurological disorders, public confidence-and therefore uptake-has been increasing steadily since 1978. Hence it is essential that the efficacy and reactogenicity of component preparations are fully evaluated in rigorous clinical trials before they are recommended in place of current British whole cell vaccines.

ElizabeTh MilleR

Principal Microbiologist,

Public Health Laboratory Service

Communicable Disease Surveillance Centre, London NW9 5EQ 
1 Sato Y, Kimura M, Fukumi H. Development of a pertussis component vaccine in Japan. Lancet $1984 ; i: 122-6$.

2 Robinson A, Irons LI, Ashworth LAE. Pertussis vaccine: present status and future prospects. Vaccine 1985;3:11-22.

3 Ashworth LAE, Fitzgeorge RB, Irons LI, Morgan CP, Robinson A. Rabbit nasopharyngeal colonisation by Bordetella pertussis: the effects of immunisation on clearance and on serum and colonisation by Bordetella pertussis: the effects of 1 C

4 Medical Research Council. Vaccination against whooping-cough. Relation between protection in children and results of laboratory tests. BrMed f 1956;ii:454-62.

5 Public Health Laboratory Service. Efficacy of whooping-cough vaccines used in the United Kingdom before 1968. Final report. BrMed f 1973;i:259-62.

6 Ashworth LAE, Robinson A, Irons LI, Morgan CP, Isaacs D. Antigens in whooping cough vaccine and antibody levels induced by vaccination of children. Lancet 1983;ii:878-81.

Romanus V, Jonsell R, Bergquist S-O, Bottiger M, Sandzelius G. Behov finns av ett vaccin mot pertussis. Läkartidningen 1984;21:2163-8.

Waight PA, Pollock TM, Miller E, Coleman EM. Pyrexia after diphtheria tetanus pertussis and diphtheria tetanus vaccines. Arch Dis Child 1983;58:921-3.

9 Pollock TM, Miller E, Mortimer JY, Smith G. Symptoms after primary immunisation with DTP and with DT vaccine. Lancet 1984;ii:146-9.

10 Miller DL, Ross EM, Alderslade R, Bellman MH, Rawson NSB. Pertussis immunisation and serious acute neurological illness in children. Br Med f 1981;282:1595-9.

11 Pollock TM Morris J. A 7-year survey of disorders atuributed to vaccination in North West Thames region. Lancet 1983; i: $753-7$.

12 Cody CL, Baraff LJ, Cherry JD, Marcy SM, Manclarke CR. Nature and rates of adverse reactions associated with DTP and DT immunisation in infants and children. Pediatrics 1981;68:650-60.

13 Anonymous. Efficacy of pertussis vaccination in England. Report from the PHLS Epidemiological Research Laboratory and 21 area health authorities. Br Med f 1982;285:357-9.

4 Preston NW. Change in serotype of pertussis infection in Britain. Lancet 1985;i:510

15 Fine PE, Clarkson JA. The recurrence of whooping cough; possible implications for assessment of vaccine efficacy. Lancet 1982;i:666-8

\section{Mouth to mask respiration}

In the absence of any equipment for cardiopulmonary resuscitation, rescuers have to use either mouth to mouth or mouth to nose expired air respiration. In practice, however, these methods may well be unacceptable to the operator should there be any blood, vomit, debris, or signs of infection in the upper airways. Similarly, fear of AIDS (the acquired immune deficiency syndrome) may well deter rescuers, even though there is no evidence of its transmission in saliva.

When basic resuscitation equipment is available (usually in the form of an oropharyngeal airway and a self inflating bag-valve-mask) it is generally not easy to use without extensive training and practice. Inexperienced single operators using a bag-valve-mask commonly find difficulty in maintaining both an airtight seal between the mask and the face and an unobstructed airway with only one hand. ${ }^{1}$ Nurses, who are generally the first on the scene of a hospital emergency, tend to find this particularly difficult. Indeed, in one study of 320 emergency medical technicians more than half were unable to adequately ventilate a manikin using a bag-valve-mask. ${ }^{2}$ Mask design also influences performance. ${ }^{23}$ Indeed, when using a bag-valve-mask two resuscitators may well be needed-one to hold the mask on to the face and to maintain a clear airway (using both hands), and the other to squeeze the bag. ${ }^{4}$

Equipment for intubating the trachea is occasionally available outside the hospital environment, since many ambulance crews now carry laryngoscopes and cuffed endotracheal tubes. Intubation is not without its hazards, however, and is certainly not a manoeuvre for the inexperienced. ${ }^{5}$

With all these difficulties, one current suggestion is that when a suitable facemask is available then mouth to mask expired air respiration ${ }^{6-9}$ should be the method of first choice for inexperienced operators. ${ }^{67}$

Mouth to mask expired air respiration was described and investigated over 30 years ago and shown to be highly effective,$^{10}$ so it is surprising that the technique is so little known. Few texts on resuscitation even mention it. The main features of the technique are, firstly, that both hands are free to hold the mask on to the face, making it much easier to achieve an airtight seal; secondly, that the fingers of both hands may be used to manipulate the lower jaw, forward if necessary, in order to maintain a clear airway; thirdly, that the operator's mouth does not come into direct contact with the patient; and, fourthly, that the operator is at the head end of the patient and is therefore well positioned to assess chest expansion during inflation.

The superiority of mouth to mask expired air respiration over bag-valve-mask resuscitation in unskilled hands is related not only to the improved seal between the mask and the face but also to the large reserve volume of air in the resuscitator's lungs. In one study of unskilled rescuers using a standard resuscitation manikin, the mean tidal volume generated by mouth to mask expired air respiration was the same as that with endotracheal intubation (about 1 litre).$^{6} \mathrm{~A}$ similar value for mouth to mask expired air respiration was found by Elling and Politis. ${ }^{2}$ The mean tidal volume for bagvalve-mask ventilation in both of these studies was well below the minimum value of $800 \mathrm{ml}$ recommended by the American Heart Association.

Ideally, masks specifically intended for use in mouth to mask expired air respiration should have a valved oxygen inlet $^{68}$ as well as other characteristics. ${ }^{7}$ In tests using the Laerdal pocket mask a mean fractional inspired oxygen concentration of $50 \%$ was achieved with expired air respiration at a rate of 12 inflations a minute (tidal volume 1 litre) using an oxygen flow of 10 litres a minute. ${ }^{8}$ Although oxygen enrichment is important, however, it does not appear to be as critical as delivery of an adequate tidal volume. ${ }^{11}$

Mouth to mask expired air respiration is a simple and effective technique and should be more widely included in resuscitation teaching programmes, particularly for those who are likely to have access to facemasks and oropharyngeal airways-for example, hospital staff, general practitioners, and accident and emergency personnel. Indeed, a strong case may be made for including a comprehensive range of both oropharyngeal airways and suitable facemasks in even very basic first aid kits. A description of this technique could then usefully be included in the Resuscitation Council's handbook Resuscitation for the Citizen. ${ }^{12}$

R W D NICKALLS Senior registrar in anaesthetics

CW Thomson Consultant anaesthetist

Department of Anaesthetics,

Newcastle General Hospital,

Newcastle upon Tyne NE4 6BE

Correspondence to: Dr Nickalls.

1 Barcroft J. Resuscitation skills. Anaesthesia 1985;40:1015-6. ventilation devices. Ann Emerg Med 1983:12:765-8.

Stewart RD, Kaplan R, Pennock B, Thompson F. Influence of mask design on bag-mask ventilation. Ann Emerg Med 1985;14:403-6.

Jesudian MCS, Harrison RR, Keenan RL, Maull KI. Bag-valve-mask ventilation; two rescuers are better than one: a preliminary report. Crit Care Med 1985;13:122-3.

Scott DB. Endotracheal intubation: friend or foe. $\mathrm{Br}$ Med 7 1986;292:157-8.

6 Harrison RR, Maull KI, Keenan RL, Boyan CP. Mouth-to-mask ventilation: a superior method of rescue breathing. Ann Emerg Med 1982;11:74-6.

American Heart Association. Standards and guidelines for cariopulmonary resuscitation (CPR) and emergency cardiac care (ECC). Part iv-advanced cardiac life support. JAMA 1980;244: 479-94.

8 Safar P. Pocket mask for emergency artificial ventilation and oxvgen inhalation. Crit Care Med 1974;2:273-6.

Safar P Cardiopulmonary cerebral resuscitation Stavanger, Norway: Asmund S Laerdal, 1981: O 62-4.

10 Elam JO, Brown ES, Elder JD. Artificial respiration by mouth-to-mask method. A study of the respiratory gas exchange of paralysed patients ventilated by operator's expired air. $N$ Engl $\mathcal{F}$ Med 1954;250:749-54.

11 Maull KI. Pocket mask ventilation: a critical reappraisal. Arch Emerg Med 1984;3:161-3.

12 Resuscitation Council (UK). Resuscitation for the citizen. London: Resuscitation Council (UK), 1984 